\title{
Childhood Attention-Deficit/Hyperactivity Disorder: A Research Review
}

\author{
Zejia $\mathrm{Cao}^{1, \uparrow, *}$, Sifan $\mathrm{Li}^{2, \dagger, *}$, Chunjie Zhang ${ }^{3, \dagger, *}$ \\ ${ }^{I}$ Faculty of Arts, University of Manitoba, Winnipeg, Manitoba R3T 2N2, Canada; \\ ${ }^{2}$ Falculty of Arts, University of British Columbia, Vancouver, British Columbia V6T 1Z4, Canada; \\ ${ }^{3}$ International Department, Shenzhen Middle School, Shenzhen, Guangdong 518001, China. \\ ${ }^{*}$ Corresponding Email: guanghua.ren@gecacademy.cn
}

These authors contributed equally.

\begin{abstract}
Attention-deficit hyperactivity disorder (ADHD) belongs to the category of neurodevelopmental disorders. The morbidity among worldwide school-aged children is $5 \%$, and $30 \%$ to $50 \%$ of it would continue to be influenced by the subsequent syndrome. The importance of exploring ADHD is very obvious and vital: to attract people's attention to ADHD for better understanding and care for this minor population of ADHD children. Three aspects were explored and elaborated on in this research: etiology, treatment, and impact. In etiology, biological and environmental factors are mentioned. The treatment methods include both pharmacological aspects, which brings up stimulant and non-stimulant medication, and the non-pharmacological aspect, which considers behavioral parenting strategies and cognitive behavioral therapy. As for impact, we focus on how ADHD affects children's social interaction from the perspective of individuals, family, and society. We hope to provide general information on ADHD in helping children with ADHD better adapt and live in this society.
\end{abstract}

Keywords: Attention-Deficit/Hyperactivity Disorder (ADHD), Children, Etiology, Impacts, Treatments

\section{INTRODUCTION}

Neurodevelopment disorder is a mental disorder that happens in the development period, mostly considered in the early stage or before school age [1]. As one of the largest mental disorder groups, it included many different early-ages developmental limitation, and one of the most common but serious ones is attention deficit hyperactivity disorder (ADHD). ADHD is an early staged generalized mental disorder characterized by impulsivity, inattentiveness, and hyperactivity that developmentally abnormal [1]. According to the research, there is a $5 \%$ prevalence worldwide among school-aged children [2], and $30 \%$ to $50 \%$ of them tend to continue to experience subsequent symptoms of the disorder in adulthood [3]. It can be a severe mental disorder and needs society to pay more attention to since it will cause a person's lifetime emotional instability, lack of cognitive memory, and attention ability. Besides, they may also suffer from highly probable comorbidities like specific learning disabilities, personality disorders, especially antisocial, obsessive-compulsive disorder, and autism [1].
Furthermore, with the seriousness of aging, more disability in the young or children means the less people take responsibility for social development, it may finally lead to a lack of social labor. Thus, this study will analyze ADHD from its distinguishes, symptom and treatment, and increase people's knowledge, understanding, and attention on ADHD.

\section{METHODOLOGY}

This research paper is conducted by searching for past published papers, mainly on authoritative medical journals and government websites. We put the keyword "Attention-deficit hyperactivity disorder" into the google scholar database and limited the scope into etiologies, treatments, and impacts of ADHD. By doing so, we can take a close look at official explanations of the cause of ADHD and further suggestions on treating ADHD children and the influence on the perspective of financial, physical, and academic stress. 


\section{THE ETIOLOGY, IMPACTS, AND TREATMENTS OF ADHD}

\subsection{Etiology of ADHD}

Potential risk factors of ADHD can be considered in terms of biological and environmental factors. A growing body of evidence suggests that genetic factor plays an important role in the process of ADHD formation [4]. The utility of inhibitory control ability is one of the genetic risks in ADHD. Motor response inhibition is a critical executive function activated when one attempts to withhold or interrupt an ongoing or planned response when external circumstances or changes in intention require it [5]. A deficiency in motor response inhibition has been identified as a risk factor for ADHD in families, with paternal ability having a greater influence on children's inhibitory control ability than maternal ability [5]. However, mothers' symptoms predict daughters' symptoms, implying girls may be more susceptible to inheriting ADHD through the maternal line [6]. In both generations, parents' inhibitory control ability considerably predicted their children's ability, regardless of symptom severity or diagnosis [5]. Consistent with research on the influence of genetic factors on the disorder, researchers found that identical twins have a higher rate of co-occurring ADHD compared to heterozygous twins [7]. Researchers' new view about ADHD is that there may be a breakdown in the brain's executive control functions [8], including focusing attention and inhibiting impulsive behavior. MRI-based studies have found abnormalities in the prefrontal cortex (PFC) in parts of the brain of children with ADHD [9]. However, PFC doesn't play an individual role in children with ADHD. Caudate and cerebellum are also tested as primary areas showing deficits in ADHD [10]. A network of neurons connects these areas and collectively control attention, thoughts, emotions, behaviors, and actions [10]. In ADHD patients, studies have revealed a slower maturation of the PFC or a reduced volume and activity of the PFC, caudate, or cerebellum [10]. In addition, ADHD has significant correlations with four genes: the dopamine D4 and D5 receptors and the dopamine and serotonin transporters [11]. Nevertheless, researchers have found that no single gene plays a major role, which means they have weak effects on ADHD if considered individually [11].

The environmental factors that have significant associations with ADHD include maternal substance use [12], stress during pregnancy [13], and family situations. Maternal substance use such as maternal smoking and alcohol abuse is likely to raise the possibility of children with ADHD [14]. Numerous studies demonstrate that maternal stress during pregnancy has a profound effect on the offspring. It has been demonstrated that when the sympathetic nervous system is activated during times of stress, an increase in uterine artery resistance occurs, resulting in decreased blood flow to the fetus [15]. This reduction in blood flow may have a detrimental effect on the development of organs, including the brain [15]. It has been shown in the literature that prenatally tressed animals' offspring experience impaired learning, cognitive function, and effects [15]. Different family situations such as serious family conflict [16] and adverse family [17] significantly correlate with childhood ADHD. At the same time, parenting styles also play a crucial role for children with ADHD. Some studies have found that maternal insensitivity, intrusive parenting, and negative affect during childhood or preschool are predictors of hyperactive and aggressive behaviors in school-aged children [18]. Recently, researchers found that some symptoms of ADHD are correlated with children exposed to toxins such as organic pollutants and lead [14]. Furthermore, various studies have assessed the efficacy of food on childhood ADHD that artificial food coloring may cause ADHD in some children [19].

It should be noted that genetic and environmental factors are not acting alone. We need to consider the role of factors and the interaction of genetic and environmental factors in developing ADHD. A recent systematic literature review concluded that differential susceptibility theory is one of the perspectives on geneenvironment interactions [20], which means certain genetic variants increase the susceptibility of individuals of ADHD [21]. In other words, children who have high susceptibility are likely to develop better in a positive environment; otherwise, they may develop worse in a negative environment. Several genes include the dopamine receptor D4 (DRD4), serotonin transporter (SLC6A4/5-HTT), and dopamine transporter gene (3SLC6A3 or DAT1), do have impacts on children with ADHD [20]. The 7-repeat DRD4 allele is considered a vulnerability factor, which means, children who carry the 7-repeat DRD4 allele are likely to be affected by poor environmental conditions [22]. In addition, serotonin transporter can moderate the effects of irritative or stressful events during early adulthood [22]. However, if children who carry short alleles and a decreasing number of serotonin transporter are apt to have strong susceptibility. Although the DAT1 gene has been investigated less than the previous two genes, the DAT1 gene has been proved that polymorphisms of the DAT1 gene have a strong correlation with children's susceptibility towards the environment [22]. Thus, children who carry DRD4, 5-HTT, or DAT1 are likely to be affected by environmental factors such as maternal expressed emotion and poor environmental conditions.

\subsection{Impacts of $A D H D$}

Children's inattentiveness is a normal phenomenon in early childhood. Still, the signs of ADHD may begin before the age of 12 years and include inappropriate development such as inattention, excessive physical 
activity, and impulsivity [23]. In addition to inattention, patients with ADHD are likely to exhibit subsequent behavioral issues such as bullying, losing one's temper, and lacking reaction to punishment. Some ADHD patients have only attention problems, while others act mainly hyperactive or impulsive behaviors. Some individuals will show a combination of attention deficit and hyperactive/impulsive problem behaviors.

ADHD children have increased difficulties in school, having a higher risk of school expulsion and academic underperformance [24]. Furthermore, some ADHD children are likely to show oppositional defiant disorder (ODD), conduct disorder (CD), and conflict with family [24]. Researchers have found that children with ADHD often lack empathy or are unable to understand the feelings of others [25]. Therefore, children with ADHD are often unpopular with their peers and are more likely to be rejected than other children [26]. Finally, Patients with ADHD are more likely to experience substance abuse in adolescence and early adulthood than children of the same age [26].

Since humans are strongly connected with the population and cannot isolate from society, the impact of ADHD can show on individuals and their family and the society they are living in. They may have inappropriate development for individuals such as inattention, excessive physical activity, and impulsivity [23], which may cause their higher risk of school expulsion and academic underperformance [24]. Focus on one or multi tasks will be harder for them than other children. They need to put more effect than others on the same task. However, most of the time, they will even challenge putting effect in one thing [1]. Furthermore, children with ADHD are more likely to experience substance abuse in adolescence and early adulthood than children of the same age because of the high risk of anti-social personality [26]. They may also higher risk in health situations like obesity.

The impacts on the family are mostly focusing on the financial, physical, and mental stress of ADHD children's family members. The family needs to bear the financial pressure under that situation for the countries that do not cover mental disease in the insurance or social welfare. They also need to be the caregiver of the children with ADHD. Both of their physical and psychological pressure will also increase. They will have a higher risk of developing some chronic diseases or mental depression because of overexertion.

Based on the features for distinguishing ADHD, criteria $\mathrm{B}$ mentioned that several symptoms for ADHD were present before 12 years old [1]. In that case, the society for them is mostly school. Their difficulties in school work will impact their academic performance and increase the difficulties in school [24]. To protect every students' right to learning, their teacher may need to pay more attention to them, and the school may also create some special programs for them. Under this situation, the working stress of school will increase.

In addition to inattention, children who have ADHD are likely to exhibit subsequent behavioral issues such as bullying, losing one's temper, and lacking reaction to punishment which will largely impact their social life. Researchers have found that children with ADHD often lack empathy or are unable to understand the feelings of others [25]. Therefore, children with ADHD are often unpopular with their peers and are more likely to be rejected than other children in school [26]. Besides, some ADHD patients have only attention problems, while others act mainly hyperactive or impulsive behaviors. Some individuals will show a combination of attention deficit and hyperactive/ impulsive problem behaviors. This combination of symptoms can also show that they will have a higher impact on society, receiving a higher risk in peer ignoring and peer bullying or violence.

\subsection{Treatments of ADHD}

While there is no remedy for ADHD at this time, there are pharmacological and non-pharmacological treatment options for symptom control and function improvement. Pharmacological treatments include stimulant and nonstimulant choices, while non-pharmacological therapies include behavioral parenting and cognitive behavioral therapy [12].

Psychostimulant, such as Methylphenidate (MPH), is widely accepted as the first-line medical treatment for ADHD in children and adolescents [27]. Stimulant medications can reduce disruptive and extremely active behaviors while expanding the attention span and calming emotions of children with ADHD. Although there is much controversy about the use of stimulant medications for ADHD, these medications help many children with ADHD calm down and focus on tasks and school for perhaps the first time in their lives. In addition, according to Buitelaar and Medori, by taking medication properly, the quality of children's socialization and selfesteem are more likely to increase because of the wellcontrolled symptoms [27]. Thus, with the sustaining medications taken, children with ADHD hope to have easier and promising lives.

As a non-stimulant medication, atomoxetine (ATX) is also served in ADHD treatment for its high bioavailability, ranging from $63 \%-94 \%$ [28]. Since both norepinephrine and dopamine are assumed to contribute to the pathophysiology of ADHD, ATX is anticipated to work in inhibiting the transporter of the presynaptic norepinephrine in the process of treating ADHD symptoms. While ATX seems to be more effective than placebo in treating ADHD, it is still not working as effectively as MPH [12].

However, no matter how effective medications are in treating ADHD, they are still limited in remitting the 
symptoms but not teaching new skills to children with ADHD, which is more sustainable and crucial for children's future development. Therefore, psychological interventions are needed to help patients with ADHD to develop more adaptive behaviors. As the most common and harmless practice, behavior therapy is mostly used to improve ADHA patients' abnormal behaviors and strengthen their ability to adapt to regular social life. To be more specific, parent training and cognitive behavioral therapy are mentioned in this paper.

Parent training is a behavior therapy intervention that aims to teach the parents to practice behavior therapy techniques with their children [29]. It is based on social learning theory. Parents are encouraged to use behavior management principles to eliminate or ignore unwanted behavior [30]. The purpose of parent training is to improve the ability and confidence of parenting behaviors and the parent-child relationship to enhance children's performance. In this case, parent training does not indicate bad parenting but aims to "optimize parenting skills to meet the above-average parenting needs of children with ADHD" [29].

Cognitive-behavioral therapy aims to help children establish a more planned and reflective way of thinking [29]. For instance, cognitive therapists help ADHD children to learn "temporary thinking", which is supposed to allow children to have rational and effective deep thinking before they appear indignation and aggressive behaviors. In addition, in the study of Miranda and Jesús Presentación, "two intervention programs of a cognitive-behavioral orientation" have considerable impact in improving "school problems and antisocial behavior" [31]. These methods used in cognitive behavioral therapy are proved to improve children's performance to better adapt social norms and receive quality life.

\section{CONCLUSION}

ADHD is a complex disorder related to multiple issues resulting in a poor quality of life. The etiology of ADHD interplay between genetic and environmental factors as heredity and susceptibility. Several genes such as the dopamine D4 and serotonin transporters are verified as potential risk factors of childhood ADHD. Besides these, deficiency of caudate and cerebellum has a significant correlation with child ADHD. Some medications such as methylphenidate (stimulants) and atomoxetine (non-stimulants) can help children correct these imbalances. Based on the current studies, cognitive behavioral therapy and parents' interventions seem to positively impact children with ADHD than medications interventions.

The limitations of this study include three aspects: etiology, sample, and treatments. Current research doesn't have enough evidence to identify the specific genes that cause children having ADHD in childhood. Most genes have strong interplay with environmental factors, but individual gene lacks studying. For future research, studying an individual gene that causes childhood ADHD is the majority purpose. It may benefit future diagnosis and treatment. In addition, the sample of the current study may not illustrate the gender difference and cultural factors. On the one hand, the current studies didn't present the gender difference in childhood ADHD, but it cannot be denied that gender difference does have different impacts on childhood ADHD. On the other hand, the majority sample of this research is collected from western culture, which means the study may not satisfy the people from eastern culture. For future studies, the sample will become more comprehensive, and a complete sample can help extend the present findings. Finally, the current study may not have enough examples to prove the accuracy and practicability of different interventions, which means the future study will deeply excavate different types of interventions.

\section{REFEREENCES}

[1] Diagnostic and statistical manual of mental disorders: DSM-5. Arlinton, VA: American Psychiatric Association, 2017.

[2] G. Polanczyk, P. Jensen, Epidemiologic Considerations in Attention Deficit Hyperactivity Disorder: A Review and Update. Child and Adolescent Psychiatric Clinics of North America, 2008 , pp. $245-260$ https://doi.org/10.1016/j.chc.2007.11.006

[3] E. Doernberg, E. Hollander, Neurodevelopmental Disorders (ASD and ADHD): DSM-5, ICD-10, and ICD-11. CNS Spectrums, 2016, pp. 295-299.

[4] M. Klein, M. Onnink, M. van Donkelaar, T. Wolfers, B. Harich, Y. Shi, J. Dammers, A. Arias-Vásquez, M. Hoogman, B. Franke, Brain imaging genetics in ADHD and beyond - Mapping pathways from gene to disorder at different levels of complexity. Neuroscience and Biobehavioral Reviews, 2017, pp. 115-155

[5] L. Goos, J. Crosbie, S. Payne, R. Schachar, Validation and Extension of the Endophenotype Model in ADHD Patterns of Inheritance in a Family Study of Inhibitory Control. The American Journal of Psychiatry, 2009, pp. 711-717. https://doi.org/10/1176/appi.aip.2009.08040621

[6] D. Steiin, J. Richards, A. Oerlemans, S. Ruiter, M. van Aken, B. Franke, J. Buitelaar, N. LambregtsRommelse, The co-occurrence of autism spectrum disorder and attention-deficit/hyperactivity disorder symptoms in parents of children with ASD or ASD with ADHD. Journal of Child Psychology and 
Psychiatry, 2012, pp. 954-963. https://doi.org/10.1111/j.1469-7610.2012.02556.x

[7] O. Grimm, S. Kittel-Schneider, A. Reif, Recent developments in the genetics of attention-deficit hyperactivity disorder: Recent developments in genetics of ADHD. Psychiatry and Clinical Neurosciences, 2018, pp. 654-672. https://doi.org/10.1111/pcn.12673

[8] A. Yasumura, M. Omori, A. Fukuda, J. Takahashi, Y. Yasumura, E. Nakagawa, T. Koike, Y. Yamashita, T. Miyajima, T. Kodea, M. Aihara, Best Abstract Award Runner-up. Predicting children with ADHD using prefrontal cortex activity. Clinical Neurophysiology, 2019, pp. e180-e181. https://doi.org/10.1016/j.clinph.2019.06.044

[9] L. Liu, Q. Zhao, X. Yu, D. Xu, H. Li, N. Ji, Z. Wu, J. Cheng, Y. Su, Q. Cao, L. Sun, Q. Qian, Y. Wang, Monoaminergic Genetic Variants, Prefrontal Cortex Amygdala Circuit, and Emotional Symptoms in Children With ADHD: Exploration Based on the Gene-Brain-Behavior Relationship. Journal of Attention Disorders, 10870547198978381087054719897838. 2020. https://doi.org/10.1177/1087054719897838

[10] A. Sharma, \& J. Couture, A review of the pathophysiology, etiology, and treatment of attention-deficit hyperactivity disorder (ADHD). The Annals of pharmacotherapy, 2014, pp. 209-225. https://doi.org/ 10.1177/1060028013510699

[11] G. Tripp, J. Wickens, Neurobiology of ADHD. Neuropharmacology, 2009, pp. 579-589. https://doi.org/10/1016/j.neuropharm.2009.07.026

[12] J. Tarver, D. Delay, K. Saval, Attention-deficit hyperactivity disorder (ADHD): an updated review of the essential facts. Child: Care, Health \& Development, 2014, pp. 762-774. https://doi.org/10/1111/cch.12139

[13] E. Sciberras, M. Mulraney, D. Silva, D. Coghill, Prenatal Risk Factors and the Etiology of ADHD Review of Existing Evidence. Current Psychiatry Reports, 2017, pp. 1-8. https://doi.org/10.1007/s11920-017-0753-2

[14] A. Thapar, M. Cooper, O. Eyre, K. Langley, Practitioner Review: What have we learnt about the causes of ADHD? Journal of Child Psychology and Psychiatry, 2013, pp. 3-16. https://doi.org/10.1111/j.1469-7610.2012.02611.x

[15] N. Grizenko, Y. Shavan, A. Polotskaia, M. TerStepanian, R. Joober, Relation of maternal stress during pregnancy to symptom severity and response to treatment in children with ADHD. Journal of psychiatry \& neuroscience, 2008, pp. 10-16.

[16] T. Banerjee, F. Middleton, S. Faraone, Environmental risk factors for attention-deficit hyperactivity disorder. Acta Pædiatrica, 2007, pp. 1269-1274. https://doi.org/10.1111/j.16512227.2007.00430.x

[17] C. Johnston, E.J. Mash, Families of children with attention-deficit/hyperactivity disorder: review and recommendations for future research. Clinical child and family psychology review, 2001, pp. 183-207. https://doi.org/10/1023/a:1017592030434

[18] C. Seipp, C. Johnston, Mother-Son Interactions in Families of Boys With AttentionDeficit/Hyperactivity Disorder With and Without Oppositional Behavior. Journal of Abnormal Child Psychology, 2005, pp. 87-98. https://doi.org/10.1007/s10802-005-0936-x

[19] J. Nigg, Future Directions in ADHD Etiology Research. Journal of Clinical Child and Adolescent Psychology, 2012, pp. 524-533. https://doi.org/10.1080/15374416.2012.686870

[20] J. Richards, C. Hartman, B. Franke, P. Hoekstra, D. Heslenfeld, J. Oosterlaan, A. Arias Vasquez, J. Buitelaar, Differential susceptibility to maternal expressed emotion in children with ADHD and their siblings? Investigating plasticity genes, prosoical and antisocial behaviour. European Child \& Adolescent Psychiatry, 2015, pp. 209-217. http://doi.org/10.1007/s00787-014-0567-2

[21] A. Wermter, M. Laucht, B. Schimmelmann, T. Banascheweski, E. Sonuga-Brake, M. Rietschel, K. Becker, From nature versus nurture, via nature and nurture, to gene $\mathrm{x}$ environment interaction in mental disorders. European Child \& Adolescent Psychiatry, 2010, pp. 199-210. https://doi.org/10.1007/s00787009-0082-z

[22] J. Belsky, M. Pluess, Beyond Diathesis Stress: Differential Susceptibility to Environmental Influences. Psychological Bulletin, 2009, pp. 885908. https://doi.org/10.1037/a0017376

[23] J. Román-Ithier, R. González, M. Vélez-Pastrana, G. González-Teiera, C. Albizu-García, Attention deficit hyperactivity disorder symptoms, type of offending and recidivism in a prison population: The role substance dependence. Criminal Behaviour and Mental Health, 2017, pp. 443-456 https://doi.org/10.1002/cbm.2009

[24] T. Peasgood, A. Bhardwaj, K. Biggs, J. Brazier, D. Coghill, C. Cooper, D. Daley, C. De Silva, V. Harpin, P. Hodgkins, A. Nadkarni, J. Setyawan, E. SonugaBarke, 2016. 
[25] H. Maoz, H. Gvirts, M. Sheffer, Y. Bloch, Theory of Mind and Empathy in Children With ADHD. Journal of Attention Disorders, 2019, pp. 1331-1338. https://doi.org/10.1177/1087054717710766

[26] R. Barkley, Global Issues Related to the Impact of Untreated Attention-Deficit/Hyperactivity Disorder From Childhood to Young Adulthood. Postgraduate Medicine, 2008, pp. 48-59. https://doi.org/10.3810/pgm.2008.09.1907

[27] J. Buitelaar, R. Medori, Treating attentiondeficit/hyperactivity disorder beyond symptom control alone in children and adolescents: a review of potential benefits of long-acting stimulants. European Child \& Adolescent Psychiatry, 2010, pp. 325-340. https://doi.org/10.1007/s00787-009-00561

[28] A.C. Childress, A critical appraisal of atomoxetine in the management of ADHD. Therapeutics and clinical risk management, 2016, pp. 27-39.

[29] NICE. Attention deficit hyperactivity disorder: diagnosis and management of ADHD in children, young people and adults. The British Psychological Society and the Royal College of Psychiatrists, London. 2008.

[30] M. Zwi, H. Jones, C. Thorgaard, A. York, J.A. Dennis, Parent training interventions for Attention Deficit Hyperactivity Disorder (ADHD) in children aged 5 to 18 years. Cochrane Database of Systematic Reviews, 2011.

[31] A. Miranda, M. Jesús Presentación, Efficacy of cognitive-behavioral therapy in the treatment of children with ADHD, with and without aggressiveness. Psychology in the Schools, 2000, pp. 169-182. 